\title{
Spectral efficiency of incoherent OCDMA systems
}

\author{
CHEN Zhuo, YU Chongxiu, ZHANG Qi, YU Zhihui, XU Daxiong \\ P.O.Box 163, School of Electronic Engineering, Beijing University of Posts and \\ Telecommunications, Beijing 100876, P.R.China \\ chnzhuo@yahoo.com.cn
}

\begin{abstract}
OCDMA allows multiple users to share a common optical channel simultaneously and asynchronously, and thus has many advantages, such as great system capacity, efficient bandwidth utilization, asynchronous access, secure communication, and so on.

Many studies on OCDMA techniques have been made in recent years. In direct-sequence (DS) incoherent OCDMA systems, each user is assigned a unique signature sequence (1's and 0's) for spreading and identification purposes. Through the use of encoding process, the user signal is spread in the frequency domain so that each user occupies the entire bandwidth available at all times. This results in a total bandwidth utilization. The optical medium is superbly suited to spread spectrum multiple access communications due to its extremely large bandwidth, however the spectral efficiency of OCDMA system is an important factor.
\end{abstract}

This paper analyses the spectrum efficiency of incoherent OCDMA systems, emphatically discusses several kinds of codes, compares and evaluates their performances.

Index terms---OCDMA, spectral efficiency, OCDMA code.

\section{INTRODUCTION}

As an originally electronic communication technique based on spread-spectrum modulation, code division multiple access (CDMA) has been widely in use for many years in mobile cellular systems operating in both 2G and so-called $3 \mathrm{G}$ systems. However, CDMA is still seeking for its properly position in optical communication.

In fact, much effort has been focused on the research of optical CDMA (OCDMA) techniques in recent years since single-mode optical fiber becomes preferred in high bit-rate long-haul communication links due to the low propagation loss and the large bandwidth available. While the vast bandwidth of the optical fiber medium provides high-speed point-to-point data transmission, the CDMA scheme facilitates random access to the channel in a bursty traffic environment. Thus, OCDMA provides a perfect means to combine the vast bandwidth available in direct detection 
optical fiber systems with the characteristics of spread spectrum multiplexing techniques. It allows multiple users to share a common optical channel simultaneously and asynchronously, and thus OCDMA is one class of system that has the advantages of being able to provide each user asynchronous access to the network, without strict wavelength controls, and provide a graceful degradation in performance as the number of users increases, besides its great system capacity and high communication security.

Because bandwidth is a scarce resource, spectral efficiency is an important performance measure in wireless communication systems. On the contrary, bandwidth is abundant in the optical domain. While the main motivation for using O-CDMA is not its efficiency of bandwidth utilization - the focus is rather on creating a low-cost, decentralized and simple high-speed multiple-access environment - spectral efficiency can nevertheless be one of a number of important measures considered in system design and performance. In an OCDMA network, spectral efficiency impacts the per-user communication rate, the number of users allowed on the network, and correspondingly the per-user cost of the system.

This paper attempts to analyze the spectral efficiency of incoherent OCDMA systems, emphatically discuss several kinds of codes, compare and evaluate their performances.

\section{INCOHERENT OCDMA SYSTEMS AND CODE DESCRIPTION}

In general, OCDMA systems can be divided into incoherent and coherent systems. Considering the most rigorous development utilizing readily available components and direct detection, the simplest implementation operates in the incoherent regime. On the other hand, if coherent schemes are operated, the phase information of the carrier is crucial in the dispreading, and consequently due to the nature of optical fiber transmission and its phase noise limitations, the complexity of the receiver makes the approach more difficult to realize.

In view of the nonnegativity constraint of the incoherent optical channel, conventional bipolar and related codes developed for the electrical domain cannot be directly applied. In other words, incoherent OCDMA is usually confined to the unipolar regime, because the optical domain is positive in nature; pulse subtraction cannot be realized in a straightforward manner. Additionally, zeroes do not carry energy. Therefore incoherent OCDMA may employ unipolar codes by the nature of the available codes.

In this paper, we describe codes with five terms: Temporal Length $L_{t}$, Wavelength Channels $N_{w}$, Weight $W$, Cardinality $C$ and Spectral Efficiency $\eta$, viz. $\operatorname{Code}\left(L_{\mathrm{t}}, N_{w}, W, C, \eta\right)$.

\section{SHANNON'S CAPACITY AND SPECTRAL EFFICIENCY}

As is known, Shannon's bound on capacity of the additive white Gaussian noise (AWGN) channel applies in the limit where coding and modulation of arbitrary complexity is used. For any communication system, the theoretically achieved channel capacity operating in an AWGN environment and utilizing any multiple access techniques is the same, since it does not matter whether the available signal space (time, wavelength channel and bandwidth) is divided into frequencies, 
time slots, or codes. In contrast to that, in a practically optical fiber environment, physical channels and code scheme exhibit temporal length, weight, wavelength channels and cardinality characteristics that result in different channel rate and system capacity. Thus, the spectral efficiency depends on the different OCDMA code schemes of the channel.

We define the spectral efficiency $\eta$ of an OCDMA system as the data rate per unit channel bandwidth for a specified average transmitted power and fixed bit error rate (BER) value, i.e.

\section{$\eta=$ Aggregate Information Rate/Total Spectral Bandwidth \\ $=N_{s u} R_{B} / N_{w} \Delta f_{c h}$.

Where $N_{s u}$ is the number of simultaneous users in the system, $R_{B}$ is the bit rate per user, $\Delta f_{c h}$ is the bandwidth of each wavelength channel, and $N_{w}$ is the number of wavelength channels used.

In OCDMA systems, $N_{s u}$ is approximately equivalent to the cardinality $C$ of codes and $L_{t}=\Delta f_{c h} / R_{B}$ when a practical optical pulse is considered, $L_{t}$ is the temporal length employed. Then, we rearrange eq. (1):

$$
\begin{aligned}
\eta & =N_{s u} R_{B} / N_{w} \Delta f_{c h} \\
& =C / N_{w} L_{t} .
\end{aligned}
$$

According to this expression, we can get that the spectral efficiency $\eta$ is directly proportional to the cardinality $C$, and it is correspondingly inversely proportional to the product of $N_{w}$ and $L_{t}$.

\section{DIFFERENT OCDMA CODE SCHEMES AND THEIR SPECTRAL EFFICIENCIES}

\section{i. Optical Orthogonal Codes (OOC)}

Regard to their excellent correlation properties, Optical Orthogonal $\operatorname{Codes}(O O C)$ feature the ultimate characteristics of codes for use in the unipolar optical domain. But in general, there are very few codes in the family of $O O C$ codes. Larger cardinality can be achieved by either increasing the length of the codes or decreasing the number of ones(Hamming weight).

For $\operatorname{OOC}\left(L_{t}, N_{w}, W, C, \eta\right), N_{w}=1$, given $L_{t}=N$,

then $C=\left(L_{t}-1\right) / W(W-1)$

$$
=(N-1) / W(W-1) \text {. }
$$

Putting $L_{t}=N, N_{w}=1$ and $C=(N-1) / W(W-1)$, then $\eta=C / N_{w} L_{t}$

$$
=(N-1) / N W(W-1) \text {. }
$$

\section{ii. Prime Codes}

Prime codes have been the focus of research in new codes for asynchronous sharing of a fiber-optic medium in many spreading incoherent OCDMA developments. Similar to the approaches used in frequency hopping applications in the electronic domain, the code algorithm relies on a block design where a code consists of many blocks each containing a single pulse. For any prime number $p$ a code comprises $p$ blocks of length $p$, the position of a pulse within the block 
being determined using the linear congruent operator. Hence, $p$ codes of length $p^{2}$ and weight $p$ are generated. The crosscorrelation of the prime codes is at most 2 , but their auto correlation is much worse and in certain time shifts the sidelobes can be as high as $p-1$.

For Prime $\left(L_{t}, N_{w}, W, C, \eta\right), N_{w}=1$, given $L_{t}=p^{2}, W=p$, then $C=p$.

Putting $L_{t}=L_{t}=p^{2}, N_{w}=1$ and $C=p$, then $\eta=C / N_{w} L_{t}$

$=p / p^{2}$

$=1 / p$.

\section{iii. Extended Quadratic Congruence Codes (EQC)}

Given the code comprises many blocks, the quadratic congruence operator can be employed to position a single pulse within that block. Increasing the block length, extended quadratic congruence codes $(E Q C)$ are developed featuring crosscorrelation of at most 2 and autocorrelation sidelobes of most 1.

For $E Q C\left(L_{t}, N_{w}, W, C, \eta\right), N_{w}=1$, given $L_{t}=p(2 p-1), W=p$

then $C=p-1$.

Putting $L_{t}=p(2 p-1), N_{w}=1$ and $C=p-1$, then $\eta=C / N_{w} L_{t}$

$=(p-1) / p(2 p-1)$.

\section{iv. Modpr Codes}

Modpr codes are generated by modify the prime codes. Though an $\mathrm{n}$ stage ladder network can only generate codes of weight $2^{n}$ with a symmetric distribution of ones, replacing entire blocks with zeroes, thereby breaking the symmetry whilst maintaining the correlation properties of the original codes, the original prime codes are modified.

For $\operatorname{Modpr}\left(L_{t}, N_{w}, W, C, \eta\right), N_{w}=1$, given $L_{t}=p^{2}$, then $C<p-2$.

Putting $L_{t}=p^{2}, N_{w}=1$ and $C<p-2$, then $\eta=C / N_{w} L_{t}$

$$
<(p-2) p^{2}
$$

\section{v. BMCOOC Codes}

BMCOOC codes are developed for ladder networks having crosscorrelation of at most 1 by using the block multiplexing approach based on Modpr codes. The codes have similar cardinality as the OOC codes. 
For $\operatorname{BMCOOC}\left(L_{t}, N_{w}, W, C, \eta\right), N_{w}=1$, given $L_{t}=N$,

then $C=\left(L_{t}-W\right) / W(W-1)+1$

$$
=(N-W) / W(W-1)+1 \text {. }
$$

Putting $L_{t}=N, N_{w}=1$ and $C=(N-W) / W(W-1)+1$,

then $\eta=C / N_{w} L_{t}$

$$
\begin{aligned}
& =[(N-W) / W(W-1)+1] / N \\
& =\left(N+W^{2}-2 W\right) / N W(W-1) .
\end{aligned}
$$

\section{vi. 2-D Prime-hop}

In order to circumvent the limitations of these classical time spread sequences, Two-dimensional (2-D) incoherent codes, spread along both the temporal and either the wavelength-channel or spatial axes, have received particular attention. To a time-spreading/wavelength-hopping scheme, every pulse(one) in the time spread pattern is transmitted at a different wavelength. Assuming a time spread pattern with $N_{t}$ codes and wavelength hoping pattern giving $N_{h}$ different sequences, the total number of codes in the family is $N_{t} N_{h}$, a large increase over existing codes. As a typical example, prime-hop scheme is explained in the following chapter.

Using the prime algorithm for the hopping and spreading patterns yields a family of codes designated as prime-hop. The number of hopping pattern is $p-1$ (the first hopping pattern is discarded as trivial), the number of spreading patterns is $\mathrm{p}$, giving $p(p-1)$ distinctive codes of length $p^{2}$. In addition to the basic structure of the symmetric codes, the asymmetric design makes use of different prime numbers for spreading and hopping pattern i.e. $p_{s}$ and $p_{h}$.

For Prime-hop $\left(L_{t}, N_{w}, W, C, \eta\right), N_{w}=p$, given $L_{t}=p^{2}, W=p$

then $C=p(p-1)$.

$$
\text { Putting } L_{t}=p^{2}, N_{w}=p \text { and } C=p(p-1),
$$

then $\eta=C / N_{w} L_{t}$

$$
\begin{aligned}
& =p(p-1) / p^{3} \\
& =(p-1) / p^{2} .
\end{aligned}
$$

Besides, there are many other codes being researched in incoherent OCDMA systems, such as EQC/Prime, SPR prime code, MPR prime code and so on.

\section{PERFORMANCE COMPARISON AND EVALUATION}

Using (3) and (7), we plot in Fig.1 the spectral efficiency $\eta$ against the weight $W$ used in the OOC and BMCOOC systems for a fixed $L_{t}(=961)$ and $N_{w}(=1)$. Within each curve, we fix the cardinality $C$ of codes and vary the weight $W$. As the weight $W$ is increased, Fig. 1 shows that the spectral efficiency nearly exponentially decreases to a very low level. Additionally, the spectral efficiency of $B M C O O C$ systems is higher than that of $O O C$ systems all along. 


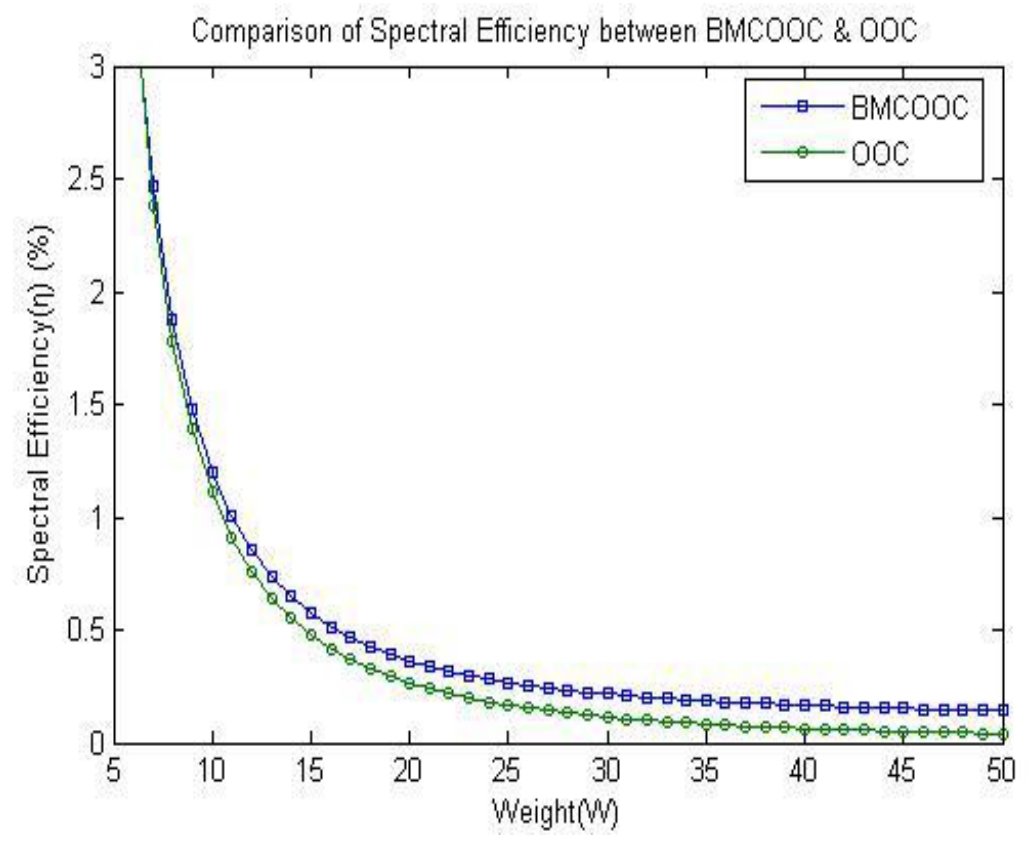

Fig.1 the spectral efficiency $\eta$ against the weight $W$ used in the $O O C$ and $B M C O O C$ systems for a fixed $L_{t}(=961)$ and $N_{w}(=1)$.

Similar to Fig.1, by (4), (5), (6) and (8) we plot $\eta$ against $W$ used in the Prime Code, Prime-hop Modpr and EQC systems for a fixed $N_{w}$ in Fig.2. The same phenomenon appears when we change the weight $W$ like we do it previous in Fig.1. And in this case, the spectral efficiency of Prime Code is the highest, and that of Prime-hop takes second place, Modpr following, and that of $E Q C$ is the lowest.

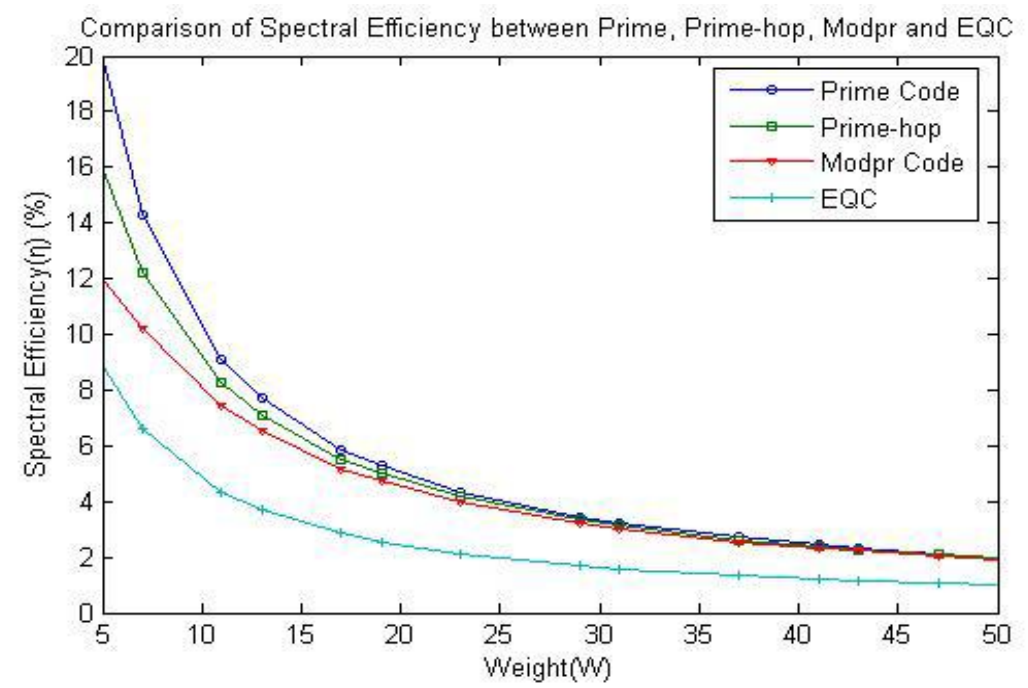

Fig.2 the spectral efficiency $\eta$ against the weight $W$ used in the Prime Code, Prime-hop Modpr and EQC systems for a fixed $N_{w}$. 
In addition, the spectral efficiencies in Fig.2 are generally superior to those in Fig.1.It is mainly because that the cardinalities of Prime Code, Prime-hop, Modpr and EQC codes are larger than those of $O O C$ and BMCOOC when their temporal length $L_{t}$ and wavelength channels $N_{w}$ are equal.

In order to gain more insight into this results in a numerical comparison, we assume some parameters for those codes above, calculate their spectral efficiencies and list them in table 1.

Table 1 Spectral Efficiencies of OCDMA Codes

\begin{tabular}{|l|l|l|l|l|l|}
\hline Code & $\begin{array}{l}\text { Temporal } \\
\text { Length }\left(L_{t}\right)\end{array}$ & $\begin{array}{l}\text { Wavelength } \\
\text { Channels }\left(N_{w}\right)\end{array}$ & Weight $(W)$ & Cardinality $(C)$ & $\begin{array}{l}\text { Spectral } \\
\text { Efficiency }(\eta)\end{array}$ \\
\hline OOC & $N=961$ & 1 & $W=8$ & $(N-1) / W(W-1)=17$ & $1.77 \%$ \\
\hline $\begin{array}{l}\text { Prime } \\
\text { Codes }\end{array}$ & $P^{2}=961$ & 1 & $W=p=31$ & $P=31$ & $3.22 \%$ \\
\hline EQC & $p(2 p-1)=1035$ & 1 & $W=p=23$ & $p-1=22$ & $2.13 \%$ \\
\hline Modpr & $N=961(p=31)$ & 1 & $W=8$ & $<p-2=27$ & $2.81 \%$ \\
\hline BMCOOC & $N=960$ & 1 & $W=8$ & $(N-W) / W(W-1)+1=18$ & $1.88 \%$ \\
\hline Prime-hop & $P^{2}=961$ & 31 & $W=p=31$ & $p(p-1)=930$ & $3.12 \%$ \\
\hline
\end{tabular}

As is shown in Fig.1 and Fig.2 and as is listed in Table 1, the spectral efficiencies of all such OCDMA systems remain very low as a whole. To a certain extent, low efficiencies militate against practical deployment of OCDMA systems in spite of their tremendous appeals. At the same time, these disappointing results have often been attributed to the unipolar(positive) nature of the optical channel. In incoherent all-optical systems, the correlation procedure incorporates non-coherent direct detection based on the power summation of optical pulses. When power, rather than a bipolar quantity such as amplitude, represents the signal, true code orthogonality, as traditionally defined, is not achievable. Therefore, research effort to improve the spectral efficiency of incoherent OCDMA systems should further be focused on the realization of pseudo-orthogonal codes and the growth of code cardinality. Furthermore, some other measures (such as Error Control Codes (ECC), optimum threshold detection asymmetric power assignment, and so on) can be taken to achieve an increase in spectral efficiency of incoherent OCDMA systems.

\section{CONCLUSIONS}

In this paper, we introduce the incoherent OCDMA systems, discuss several kinds of code schemes and analyze their expressions of spectral efficiency. The comparisons and analyses of their spectral efficiencies are given in the end.

\section{ACKNOWLEDGENT}

This work was supported by the National Science Foundation of China under Grant No.60177018. 


\section{REFERENCES}

[1] J. A. Salehi, "Code division multiple access techniques in optical fiber networks - part I: Fundamental principles," IEEE Trans. Commun., vol. 37, pp. 824-833, Aug. 1989.

[2] J. A. Salehi and C. A. Brackett, "Code division multiple access techniques in optical fiber networks-Part II: Systems performance analysis," IEEE Trans. Commun., vol. 37, pp. 834-842, Aug. 1989.

[3] I. Andonovic and L. Tancevski. "Incoherent optical code division multiple access systems," Proceedings of IEEE 4th International Symposium on Spread Spectrum Techniques and Applications, vol. 1, pp. 424-430, Sep. 1996.

[4] F. R. K. Chung, J. A. Salehi, and V. K. Wei, "Optical orthogonal codes: design, analysis, and applications," IEEE Trans. Inform. Theory, vol. 35, pp. 595-604, May 1989.

[5] Eddie K. H. Ng and Edward H. Sargent, "Mining the fibre optic channel capacity in the local area: maximizing spectral efficiency in multi wavelength optical CDMA networks," IEEE International Conference on Communications, vol. 3, pp. 712-715, June, 2001.

[6] L. Tancevski and I. Andonovic, "Wavelength hopping/time spreading code division multiple access systems," Electron. Lett., vol. 30, no. 17, pp. 1388-1390, Aug. 1994.

[7] J. G. Zhang and W. C. Kwong, "Design of optical code-division multiple- access networks with modified prime codes," in 1997 IEEE Int. Symp. on Information Theory, 1997, p. 480.

[8] E. S. Shivaleela, K. N. Sivarajan, and A. Selvarajan, "Design of a new family of two-dimensional codes for fiber-optic CDMA networks," J. Lightwave Technol., vol. 16, pp. 501-508, Apr. 1998.

[9] L. Tancevski, I. Andonovic, M. Tur, and J. Budin, "Hybrid wavelength hopping/time spreading code division multiple access systems," IEE Proc. Optoelectron., vol. 143, no. 3, pp. 161-166, 1996.

[10] T.-W. F. Chang and E. H. Sargent, "Optical CDMA using 2-D codes: the optimal single-user detector," IEEE Commun. Lett., vol. 5, pp. 169-171, Apr. 2001.

[11] T.-W. F. Chang and E. H. Sargent, "Optimizing Spectral Efficiency in Multiwavelength Optical CDMA System," IEEE Trans. on Commun., vol. 51, no. 9, pp. 1442-1445, Sep. 2003

[12] Eddie K. H. Ng and Edward H. Sargent, "Optimum Threshold Detection in Real-Time Scalable High-Speed Multi-Wavelength Optical Code-Division Multiple-Access LANs," IEEE Trans. on Commun., vol. 50, no. 5, pp. 778-784, May, 2002

[13] Tung-Wah Frederick Chang and Edward H. Sargent, "Spectral efficiency limit of bipolar signaling in incoherent optical CDMA systems," in Proc. IEEE Global Telecommunications Conf. (GLOBECOM'01), San Antonio, TX, pp. 1484-1486, Nov. 2001.

[14] Frederik Vanhaverbeke and Marc Moeneclaey, "Sum Capacity of the OCDMA/OCDMA Signature Sequence Set," IEEE Commun. Lett., vol. 6, no. 8, pp. 340-342, Aug. 2002. 